PROCEEDINGS OF THE

AMERICAN MATHEMATICAL SOCIETY

Volume 125, Number 9, September 1997, Pages 2589-2598

S $0002-9939(97) 04080-\mathrm{X}$

\title{
EXISTENCE OF TRAVELING WAVE FRONTS IN DELAYED REACTION-DIFFUSION SYSTEMS VIA THE MONOTONE ITERATION METHOD
}

\author{
XINGFU ZOU AND JIANHONG WU \\ (Communicated by Hal L. Smith)
}

\begin{abstract}
The monotone iteration method is employed to establish the existence of traveling wave fronts in delayed reaction-diffusion systems with monostable nonlinearities.
\end{abstract}

\section{Introduction AND the MAIN RESUlt}

Traveling wave solutions for reaction-diffusion (R-D) equations without delay have been extensively studied in the literature. The recent book review of Gardner [4] and the monographs of Fife [3], Britton [2], Murray [6], and Volpert, Volpert and Volpert [9] provide a full discussion of the subject.

On the other hand, time delay should be and has been incorporated into realistic models in many applications. The pioneering work of Schaaf [8] investigated the existence and stability of traveling wave fronts for several classes of delayed scalar reaction-diffusion equations. However, it seems that little has been done for systems of delayed reaction-diffusion equations.

The purpose of this paper is to tackle the existence of traveling wave front solutions of delayed $R$-D systems with monostable nonlinearities. Our approach is based on monotone iteration techniques for elliptic systems with advanced arguments (we refer to Leung [5] and Pao [7] for some related monotone iteration schemes), and our choice of the upper and lower solutions is inspired by but different from the work of Volpert, Volpert and Volpert [9] for R-D systems without delay.

Consider the following system of reaction-diffusion equations with delay:

$$
\frac{\partial u(x, t)}{\partial t}=A \frac{\partial^{2} u(x, t)}{\partial x^{2}}+f(u(x, t), u(x, t-\tau)), \quad t \geq 0, x \in \mathbb{R},
$$

where $u \in \mathbb{R}^{n}, A=\operatorname{diag}\left(a_{1}, \ldots, a_{n}\right), a_{i}>0,1 \leq i \leq n$, and $f: \mathbb{R}^{n} \times \mathbb{R}^{n} \rightarrow \mathbb{R}^{n}$ is a map satisfying the following conditions:

(A1) $f: \mathbb{R}^{n} \times \mathbb{R}^{n} \rightarrow \mathbb{R}^{n}$ is locally Lipschitz.

Received by the editors January 24, 1996.

1991 Mathematics Subject Classification. Primary 34K10, 35K10, 35K55.

Key words and phrases. Reaction-diffusion equations, traveling wave fronts, monotone iteration.

This research was partially supported by the Natural Sciences and Engineering Research Council of Canada. 
(A2) $f(\mathbf{0}, \mathbf{0})=f(\mathbf{1}, \mathbf{1})=0$, and $f(u, v)>0$ for $\mathbf{0}<u, v<\mathbf{1}$, where $\mathbf{z}=$ $(z, \ldots, z)^{T} \in \mathbb{R}^{n}$ for every $z \in \mathbb{R}$ and the ordering in $\mathbb{R}^{n}$ is defined by: $u \leq v$ for $u, v \in \mathbb{R}^{n}$ if and only if $u_{i} \leq v_{i}$ for all $1 \leq i \leq n$; and $u<v$ if and only if $u \leq v$ and $u \neq v$.

A traveling wave of (1.1) is a solution $u(x, t)$ of the form

$$
u(x, t)=w(x-c t)
$$

for some constant $c>0$ and some $w \in C^{2}\left(\mathbb{R} ; \mathbb{R}^{n}\right)$ which satisfies the elliptic system with advanced argument

$$
A w^{\prime \prime}(x)+c w^{\prime}(x)+f(w(x), w(x+c \tau))=0, \quad x \in \mathbb{R} .
$$

We will look for monotone wave fronts which are monotone traveling waves connecting the two stationary points $\mathbf{0}$ and $\mathbf{1}$ such that

$$
\lim _{x \rightarrow \infty} w(x)=\mathbf{0}, \quad \lim _{x \rightarrow-\infty} w(x)=\mathbf{1} .
$$

Let $\Gamma$ be the set of all functions $\rho \in C^{2}\left(\mathbb{R} ; \mathbb{R}^{n}\right)$ with the following properties:

(i) $\lim _{x \rightarrow \infty} \rho(x)=\mathbf{0}$ and $\lim _{x \rightarrow-\infty} \rho(x)=\mathbf{1}$;

(ii) $\rho_{j}^{\prime}(x)<0$ for all $x \in \mathbb{R}$ and $1 \leq j \leq n$.

We define a parameterized functional $\Psi$ on $\Gamma \times(0, \infty) \times[0, \infty)$ by

$$
\Psi(\rho, c, \tau)=\sup _{x \in \mathbb{R}, 1 \leq j \leq n} \frac{a_{j} \rho_{j}^{\prime \prime}(x)+f_{j}(\rho(x), \rho(x+c \tau))}{-\rho_{j}^{\prime}(x)} .
$$

Moreover, for a fixed $\tau \geq 0$ we set

$$
g_{\tau}(c)=\inf _{\rho \in \Gamma} \Psi(\rho, c, \tau) .
$$

Lemma 1.1. If, in addition to (A1) and (A2), we assume that

(A3) $f(u, v)$ is monotonically increasing in $v$ for $\mathbf{0} \leq u, v \leq \mathbf{1}$, that is, $f(u, v) \leq$ $f(u, w)$ for all $v, w \in \mathbb{R}^{n}$ with $\mathbf{0} \leq v \leq w \leq \mathbf{1}$,

then

(1) $g_{\tau}(c) \geq 0$ for any $c \geq 0$ and $\tau \geq 0$;

(2) for fixed $\tau>0$, if $g_{\tau}\left(c_{0}\right)<\infty$ for some $c_{0} \geq 0$, then $g_{\tau}(c)<\infty$ for all $c \geq c_{0}$. In this case, there exists a unique $c^{*}(\tau) \geq 0$ such that $g_{\tau}(c)<c$ for all $c>c^{*}(\tau)$.

Proof. (1) By (A2), we know that for any $\rho \in \Gamma, f_{j}(\rho(x), \rho(y))>0$ for $x, y \in \mathbb{R}$ and $1 \leq j \leq n$. Thus

$$
\Psi(\rho, c, \tau) \geq \sup _{x \in \mathbb{R}, 1 \leq j \leq n} \frac{a_{j} \rho_{j}^{\prime \prime}(x)}{-\rho_{j}^{\prime}(x)}, \quad \rho \in \Gamma .
$$

We claim that for each $1 \leq j \leq n$ there exists $x_{j} \in \mathbb{R}$ such that $\rho_{j}^{\prime \prime}\left(x_{j}\right)>0$. For otherwise, we would have $\rho_{j}^{\prime}(x) \leq \rho_{j}^{\prime}(0)<0$ for all $x \geq 0$, and hence $\rho_{j}(x) \leq$ $\rho_{j}(0)+\rho_{j}^{\prime}(0) x$ for all $x \geq 0$, which would yield $\lim _{x \rightarrow \infty} \rho_{j}(x)=-\infty$, a contradiction to the positivity of $\rho_{j}$. Thus

$$
\Psi(\rho, c, \tau) \geq \frac{a_{j} \rho_{j}^{\prime \prime}\left(x_{j}\right)}{-\rho_{j}^{\prime}\left(x_{j}\right)}>0, \quad \rho \in \Gamma,
$$

and therefore, $g_{\tau}(c) \geq 0$.

(2) (A3) ensures that $\Psi(\rho, c, \tau)$ is decreasing in $c$ for every $\rho \in \Gamma$, and hence $g_{\tau}(c)$ is also decreasing in $c$. From this and (1), we arrive at the conclusion. 
The following is the main theorem of this paper, the proof of which will be given in $\S 3$.

Theorem 1.2. Assume (A1)-(A3) are satisfied. Moreover, we assume

(A4) there exists $d \geq 0$ such that

$$
f_{i}(u, v)-f_{i}\left(u^{*}, v^{*}\right) \geq-d\left(u_{i}-u_{i}^{*}\right)
$$

$$
\text { for } i=1, \ldots, n \text { and } \mathbf{0} \leq u^{*} \leq u \leq \mathbf{1}, \mathbf{0} \leq v^{*} \leq v \leq \mathbf{1}
$$

Suppose also $g_{\tau}\left(c_{0}\right)<\infty$ for some $c_{0}>0$. Then for any $c>c^{*}(\tau)$, where $c^{*}(\tau)$ is defined in (2) of Lemma 1.1, there exists a monotonically decreasing solution of (1.3) satisfying condition (1.4) at $\pm \infty$.

\section{Monotone iteration}

In this section, we establish some monotone iteration results for elliptic systems with advanced arguments both on bounded and unbounded domains. These results will not only be used to prove the main theorem, but also be of interest on their own. We start with bounded domains and then pass to unbounded domains by taking limits. In what follows, we set $u \leq v$ for $u, v \in C\left(I ; \mathbb{R}^{n}\right)$ if and only if $u(x) \leq v(x)$ for $x \in I$, where $I$ is a subset of $\mathbb{R}$.

Let $\delta>0$ be a fixed constant. Assume $a$ and $b$ are real numbers such that $a<b-2 \delta$. Consider the following elliptic system with advanced argument:

$$
-(L u)(x)=g(u(x), u(x+\delta)), \quad x \in(a, b),
$$

and

$$
u(a)=h_{a}, \quad u(b)=h_{b},
$$

where $h_{a}, h_{b} \in \mathbb{R}^{n}$ are fixed vectors,

$$
L u=A u^{\prime \prime}+c u^{\prime}
$$

for $u \in C^{2}\left((a, b) ; \mathbb{R}^{n}\right), A=\operatorname{diag}\left(a_{1}, \ldots, a_{n}\right), a_{1}, \ldots, a_{n}$ and $c$ are positive constants, and $g: \mathbb{R}^{n} \times \mathbb{R}^{n} \rightarrow \mathbb{R}^{n}$ is a given mapping to be specified later.

Definition 2.1. By an upper solution of (2.1)-(2.2), we mean a map $\tilde{u} \in$ $C^{\alpha}\left([a, b+\delta] ; \mathbb{R}^{n}\right) \cap C^{2}\left([a, b] ; \mathbb{R}^{n}\right)$ which satisfies

$$
\left\{\begin{array}{l}
-(L \tilde{u})(x) \geq g(\tilde{u}(x), \tilde{u}(x+\delta)), \quad x \in(a, b), \\
\tilde{u}(a) \geq h_{a}, \quad \tilde{u}(b) \geq h_{b} .
\end{array}\right.
$$

Similarly, we can define lower solutions by reversing the inequalities in (2.3).

A pair of an upper solution $\tilde{u}$ and a lower solution $\hat{u}$ is called ordered if $\tilde{u} \geq \hat{u}$ in $[a, b]$. For any pair of ordered upper and lower solutions $\tilde{u} \geq \hat{u}$, we denote by $[\hat{u}, \tilde{u}]$ the sector of all functions $u \in C\left([a, b] ; \mathbb{R}^{n}\right)$ such that $\hat{u}(x) \leq u(x) \leq \tilde{u}(x)$ for $x \in[a, b]$.

We have the following monotone iteration theorem:

Theorem 2.2. Let $\tilde{u} \geq \hat{u}$ be a pair of ordered upper and lower solutions of (2.1)(2.2). Assume that $g: \mathbb{R}^{n} \times \mathbb{R}^{n} \rightarrow \mathbb{R}^{n}$ is locally Lipschitz, $g(\mathbf{0}, \mathbf{0})=\mathbf{0}$ and $g$ satisfies the following quasimonotonicity condition:

(QM) There exists $d \geq 0$ such that $g_{i}(u, v)-g_{i}\left(u^{*}, v^{*}\right) \geq-d\left(u_{i}-u_{i}^{*}\right)$ for $i=$ $1, \ldots, n$, and $u, u^{*}, v, v^{*} \in \mathbb{R}^{n}$ such that $\hat{u}(x) \leq u^{*} \leq u \leq \tilde{u}(x)$ and $\hat{u}(x) \leq v^{*} \leq v \leq \tilde{u}(x)$ for some $x \in[a, b+\delta]$. 
Then, there exist $\bar{u}, \underline{u} \in C^{2+\alpha}\left([a, b-2 \delta] ; \mathbb{R}^{n}\right) \cap C^{1+\alpha}\left([a, b] ; \mathbb{R}^{n}\right)$ such that

(1) $\hat{u}(x) \leq \underline{u}(x) \leq \bar{u} \leq \tilde{u}(x)$ for $x \in[a, b]$;

(2) both $\underline{u}$ and $\bar{u}$ satisfy (2.1) in $(a, b-2 \delta)$ and the boundary condition (2.2);

(3) $\bar{u}(\underline{u})$ is the maximal (minimal) solution in the following sense: if $u^{*} \in$ $C^{2+\alpha}\left([a, b-2 \delta] ; \mathbb{R}^{n}\right) \cap C^{1+\alpha}\left([a, b] ; \mathbb{R}^{n}\right)$ satisfies $(2.1)$ in $(a, b-2 \delta)$ and the boundary condition (2.2), and if $\hat{u} \leq u^{*} \leq \tilde{u}$ in $[a, b-2 \delta]$, then $\underline{u} \leq u^{*} \leq \bar{u}$ in $[a, b]$.

Proof. The main idea of the proof is to construct convergent sequences from the ordered pair $\tilde{u} \geq \hat{u}$.

Step 1. Construction of monotone sequences: For $\bar{v}^{(0)}:=\tilde{u} \in C^{\alpha}\left([a, b+\delta] ; \mathbb{R}^{n}\right)$, consider the boundary value problem

$$
\left\{\begin{array}{l}
-\left(L u^{(1)}\right)(x)+d u^{(1)}(x)=\bar{q}^{(0)}(x) \quad \text { in }(a, b), \\
u^{(1)}(a)=h_{a}, \quad u^{(1)}(b)=h_{b},
\end{array}\right.
$$

where

$$
\bar{q}^{(0)}(x)=d \bar{v}^{(0)}(x)+g\left(\bar{v}^{(0)}(x), \bar{v}^{(0)}(x+\delta)\right), \quad x \in[a, b] .
$$

By the well-known Schauder's theory, we can show that (2.4) has a unique solution $\bar{u}^{(1)} \in C^{2+\alpha}\left([a, b] ; \mathbb{R}^{n}\right)$.

Similarly, starting from $\underline{v}^{(0)}:=\hat{u} \in C^{\alpha}\left([a, b+\delta] ; \mathbb{R}^{n}\right)$, we can obtain a unique solution $\underline{u}^{(1)} \in C^{2+\alpha}\left([a, b] ; \mathbb{R}^{n}\right)$ of the boundary value problem

$$
\left\{\begin{array}{l}
-\left(L u^{(1)}\right)(x)+d u^{(1)}(x)=\underline{q}^{(0)}(x) \quad \text { in }(a, b), \\
u^{(1)}(a)=h_{a}, \quad u^{(1)}(b)=h_{b}
\end{array}\right.
$$

where

$$
\underline{q}^{(0)}(x)=d \underline{v}^{(0)}(x)+g\left(\underline{v}^{(0)}(x), \underline{v}^{(0)}(x+\delta)\right), \quad x \in[a, b] .
$$

Applying the maximum principle and the quasimonotonicity condition (QM), we can establish

$$
\hat{u}=\underline{v}^{(0)} \leq \underline{u}^{(1)} \leq \bar{u}^{(1)} \leq \bar{v}^{(0)}=\tilde{u} \quad \text { on }[a, b] .
$$

We now modify the above $\bar{u}^{(1)}$ and $\underline{u}^{(1)}$ to obtain $C^{\alpha}$-extensions $\bar{v}^{(1)}, \underline{v}^{(1)} \in$ $C^{\alpha}\left([a, b+\delta] ; \mathbb{R}^{n}\right)$ on $[a, b]$ such that

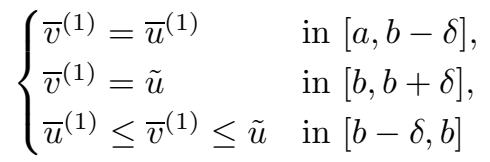

and

$$
\begin{cases}\underline{v}^{(1)}=\underline{u}^{(1)} & \text { in }[a, b-\delta], \\ \underline{v}^{(1)}=\hat{u} & \text { in }[b, b+\delta], \\ \hat{u} \leq \underline{v}^{(1)} \leq \underline{u}^{(1)} & \text { in }[b-\delta, b] .\end{cases}
$$

Clearly, we have

$$
\hat{u} \leq \underline{v}^{(1)} \leq \bar{v}^{(1)} \leq \tilde{u} \quad \text { on }[a, b+\delta] .
$$


Repeating the above procedure inductively, we can obtain two sequences of functions $\left\{\bar{u}^{(k)}\right\}_{k \geq 1}$ and $\left\{\underline{u}^{(k)}\right\}_{k \geq 1}$ on $[a, b]$, as well as their $C^{\alpha}$-extensions $\left\{\bar{v}^{(k)}\right\}_{k \geq 1}$ and $\left\{\underline{v}^{(k)}\right\}_{k \geq 1}$ on $[a, b+\delta]$, such that

$$
\left\{\begin{array}{l}
-L \bar{u}^{(k)}+d \bar{u}^{(k)}=\bar{q}^{(k-1)} \quad \text { in }(a, b), \\
\bar{u}^{(k)}(a)=h_{a}, \quad \bar{u}^{(k)}(b)=h_{b}
\end{array}\right.
$$

and

$$
\left\{\begin{array}{l}
-L \underline{u}^{(k)}+d \underline{u}^{(k)}=\underline{q}^{(k-1)} \quad \text { in }(a, b), \\
\underline{u}^{(k)}(a)=h_{a}, \quad \underline{u}^{(k)}(b)=h_{b},
\end{array}\right.
$$

where

$$
\begin{aligned}
& \bar{q}^{(k-1)}(x)=d \bar{v}^{(k-1)}(x)+g\left(\bar{v}^{(k-1)}(x), \bar{v}^{(k-1)}(x+\delta)\right), \quad x \in[a, b], \\
& \underline{q}^{(k-1)}(x)=d \underline{v}^{(k-1)}(x)+g\left(\underline{v}^{(k-1)}(x), \underline{v}^{(k-1)}(x+\delta)\right), \quad x \in[a, b],
\end{aligned}
$$

and $\bar{v}^{(k-1)}, \underline{v}^{(k-1)} \in C^{\alpha}\left([a, b+\delta] ; \mathbb{R}^{n}\right)$ satisfy

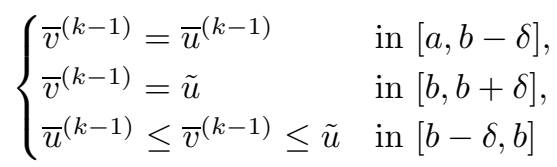

and

$$
\begin{cases}\underline{v}^{(k-1)}=\underline{u}^{(k-1)} & \text { in }[a, b-\delta] \\ \underline{v}^{(k-1)}=\hat{u} & \text { in }[b, b+\delta] \\ \hat{u} \leq \underline{v}^{(k-1)} \leq \underline{u}^{(k-1)} & \text { in }[b-\delta, b]\end{cases}
$$

Moreover, we have

$$
\begin{cases}\hat{u} \leq \underline{u}^{(k-1)} \leq \underline{u}^{(k)} \leq \bar{u}^{(k)} \leq \bar{u}^{(k-1)} \leq \tilde{u} & \text { on }[a, b], \\ \hat{u} \leq \underline{v}^{(k-1)} \leq \underline{v}^{(k)} \leq \bar{v}^{(k)} \leq \bar{v}^{(k-1)} \leq \tilde{u} & \text { on }[a, b+\delta] .\end{cases}
$$

Step 2: Convergence of monotone sequences: The monotonicity implies the existence of the following limits:

$$
\lim _{k \rightarrow \infty} \bar{u}^{(k)}=\bar{u}, \quad \lim _{k \rightarrow \infty} \underline{u}^{(k)}=\underline{u}, \quad \lim _{k \rightarrow \infty} \bar{v}^{(k)}=\bar{v}, \quad \lim _{k \rightarrow \infty} \underline{v}^{(k)}=\underline{v} .
$$

Obviously, $\bar{v}=\bar{u}$ and $\underline{v}=\underline{u}$ on $[a, b-\delta]$. Moreover, $\underline{u} \leq \bar{u}$ on $[a, b]$. We wish to show that $\bar{u}$ and $\underline{u}$ satisfy $(2.1)$ in $(a, b-2 \delta)$ and the boundary condition (2.2).

By the continuity of $g$, we know that

$$
\bar{q}^{(k)}(x) \rightarrow d \bar{v}(x)+g(\bar{v}(x), \bar{v}(x+\delta)) \quad \text { uniformly for } x \in[a, b] \text { as } n \rightarrow \infty .
$$

Therefore, the sequence $\left\{\bar{q}^{(k)}\right\}_{k \geq 1}$ is bounded in $L_{p}\left([a, b] ; \mathbb{R}^{n}\right)$ for each $p \geq 1$, and hence $\left\{\bar{u}^{(k)}\right\}_{k \geq 1}$ is bounded in $W_{p}^{2}\left([a, b] ; \mathbb{R}^{n}\right)$, by Agmon-Douglis-Nirenberg's boundary $L_{p}$-estimate (see, for example, Agmon, Douglis and Nirenberg [1]). It follows from the standard embedding theorem that $\left\{\bar{u}^{(k)}\right\}_{k \geq 1}$ is bounded in $C^{1+\mu}\left([a, b] ; \mathbb{R}^{n}\right)$ where $\mu=1-\frac{1}{p}$. Now we choose sufficiently large $p$ such that $\mu=1-\frac{1}{p}>\alpha$. Then there exists a constant $M>0$ such that

$$
\left\|\bar{u}^{(k)}\right\|_{C^{1+\alpha}\left([a, b] ; \mathbb{R}^{n}\right)} \leq\left\|\bar{u}^{(k)}\right\|_{C^{1+\mu}\left([a, b] ; \mathbb{R}^{n}\right)} \leq M, \quad k \geq 1 .
$$


The Arzela-Ascoli theorem implies that $\left\{\bar{u}^{(k)}\right\}_{k>0}$ has a subsequence which converges to a $u^{*} \in C^{1+\alpha}\left([a, b] ; \mathbb{R}^{n}\right)$ in $C^{1}\left([a, b] ; \mathbb{R}^{n}\right)$. This, coupled with the established pointwise convergence in $[a, b]$, implies that the whole sequence $\left\{\bar{u}^{(k)}\right\}_{k \geq 0}$ converges to $\bar{u}=u^{*} \in C^{1+\alpha}\left([a, b] ; \mathbb{R}^{n}\right)$ in $C^{1}\left([a, b] ; \mathbb{R}^{n}\right)$.

Next, we shrink the interval to $[a, b-2 \delta]$ to obtain estimates of higher order derivatives. Indeed, by the standard Schauder's estimate and the boundedness of the solution $\bar{u}^{(k)}$ in $C^{1+\alpha}\left([a, b] ; \mathbb{R}^{n}\right)$, we can show that $\left\{\bar{u}^{(k)}\right\}_{k \geq 1}$ is bounded in $C^{2+\alpha}\left([a, b-2 \delta] ; \mathbb{R}^{n}\right)$. Again by the Arzela-Ascoli theorem, there exists a subsequence of $\left\{\bar{u}^{(k)}\right\}_{k \geq 1}$ which converges to $u^{* *} \in C^{2+\alpha}\left([a, b-2 \delta] ; \mathbb{R}^{n}\right)$ in the $C^{2}\left([a, b-2 \delta] ; \mathbb{R}^{n}\right)$-topology. But, $\bar{u}^{(k)} \rightarrow \bar{u}$ pointwise in $[a, b]$ as $k \rightarrow \infty$. Hence, the whole sequence $\left\{\bar{u}^{(k)}\right\}_{k \geq 1}$ itself converges to $\bar{u}=u^{* *} \in C^{2+\alpha}\left([a, b-2 \delta] ; \mathbb{R}^{n}\right)$ in the $C^{2}\left([a, b-2 \delta] ; \mathbb{R}^{n}\right)$-topology. This implies that $L \bar{u}^{(k)} \rightarrow L \bar{u}$ pointwise in $[a, b-\delta]$. Taking the limit as $k \rightarrow \infty$ in (2.9), we arrive at

$$
-(L \bar{u})(x)+d \bar{u}(x)=d \bar{u}(x)+g(\bar{v}(x), \bar{v}(x+\delta)), \quad x \in[a, b-2 \delta] .
$$

Noticing that $\left.\bar{v}\right|_{[a, b-\delta]}=\bar{u}$, we finally get

$$
-(L \bar{u})(x)=g(\bar{u}(x), \bar{u}(x+\delta)), \quad x \in(a, b-2 \delta) .
$$

Similarly, we can prove $\underline{u} \in C^{2+\alpha}\left([a, b-2 \delta] ; \mathbb{R}^{n}\right) \cap C^{1+\alpha}\left([a, b] ; \mathbb{R}^{n}\right)$ and $\underline{u}$ satisfies $(2.1)$ in $(a, b-2 \delta)$ and the boundary condition (2.2).

Step 3: Maximality of $\bar{u}$ and minimality of $\underline{u}$ : Suppose $u^{*} \in C^{2+\alpha}\left([a, b-2 \delta] ; \mathbb{R}^{n}\right) \cap$ $C^{1+\alpha}\left([a, b] ; \mathbb{R}^{n}\right)$ is given so that $\hat{u} \leq u^{*} \leq \tilde{u}$ in $[a, b], u^{*}(a)=h_{a}, u^{*}(b)=h_{b}$ and $u^{*}$ satisfies $(2.1)$ in $(a, b-2 \delta)$. Choose $\hat{v} \in C^{\alpha}\left([a, b+\delta] ; \mathbb{R}^{n}\right)$ such that

(i) $\hat{v} \leq \tilde{u}$ in $[a, b+\delta]$;

(ii) $\hat{v}=u^{*}$ in $[a, b]$.

Now, we repeat Step 1 and Step 2, replacing the ordered pair $\langle\hat{u}, \tilde{u}\rangle$ by $\langle\hat{v}, \tilde{u}\rangle$, to obtain a pair of vector functions $\bar{u}^{*}, \underline{u}^{*} \in C^{2+\alpha}\left([a, b-2 \delta] ; \mathbb{R}^{n}\right) \cap C^{1+\alpha}\left([a, b] ; \mathbb{R}^{n}\right)$ satisfying $\hat{v} \leq \underline{u}^{*} \leq \bar{u}^{*} \leq \tilde{u}$ in $[a, b]$. Examining the procedure of the construction of the sequence $\left\{\bar{v}^{(k)}\right\}_{k \geq 1}$ convergent to $\bar{u}$, we find that each $\bar{v}^{(k)}$ is actually independent of the choice of the lower solution $\hat{u}$ in the ordered pair. Therefore, as the limit of the same sequence, we must have $\bar{u}^{*}=\bar{u}$ in $[a, b]$. Consequently, we have $u^{*}=\hat{v} \leq \underline{u}^{*} \leq \bar{u}^{*}=\bar{u}$ in $[a, b]$.

A similar argument leads to the claim that $\underline{u} \leq u^{*}$ in $[a, b]$. This completes the proof.

We now extend the above result to the unbounded domain

$$
-(L u)(x)=g(u(x), u(x+\delta)), \quad x \in \mathbb{R} .
$$

Definition 2.3. $\bar{\rho}$ is called an upper solution of $(2.14)$ if $\bar{\rho} \in C^{2}\left(\mathbb{R} ; \mathbb{R}^{n}\right)$ and satisfies

$$
-(L \bar{\rho})(x) \geq g(\bar{\rho}(x), \bar{\rho}(x+\delta)), \quad x \in \mathbb{R} .
$$

Similarly, we can define lower solutions of (2.14) by reversing the inequality in (2.15).

In what follows, we assume that $\bar{\rho}$ and $\rho$ are a pair of upper and lower solutions of (2.14) satisfying $\underline{\rho} \leq \bar{\rho}$ in $\mathbb{R}$. We will consider

$$
-(L u)(x)=g(u(x), u(x+\delta)), \quad x \in[-m r, m r],
$$

subject to either

$$
u( \pm m r)=\underline{\rho}( \pm m r)
$$


or

$$
u( \pm m r)=\bar{\rho}( \pm m r)
$$

where $r$ is the constant given by $r=1+2 \delta>0$.

Assume $g: \mathbb{R}^{n} \times \mathbb{R}^{n} \rightarrow \mathbb{R}^{n}$ is locally Lipschitz, $g(\mathbf{0}, \mathbf{0})=\mathbf{0}$ and satisfies the following quasimonotonicity condition:

$(\mathrm{QM})^{*}$ There exists $d \geq 0$ such that $g_{i}(u, v)-g_{i}\left(u^{*}, v^{*}\right) \geq-d\left(u_{i}-u_{i}^{*}\right)$ for $i=$ $1, \ldots, n$, and $u, u^{*}, v, v^{*} \in \mathbb{R}^{n}$ such that $\rho(x) \leq u^{*} \leq u \leq \bar{\rho}(x)$ and $\rho(x) \leq v^{*} \leq v \leq \bar{\rho}(x)$ for some $x \in \mathbb{R}$.

Let $\bar{u}_{m}$ be the maximal solution of (2.16) and (2.18) and $\underline{u}_{m}$ be the minimal solution of (2.16) and (2.17) in the sense of Theorem 2.2.

Lemma 2.4. Suppose further that $g$ satisfies the following positivity condition:

(P) $g(u, v) \geq 0$ for $u, v \in \mathbb{R}^{n}$ satisfying $\underline{\rho}(x) \leq u, v \leq \bar{\rho}(x)$ for some $x \in \mathbb{R}$.

Then, if $\bar{\rho}_{i}^{\prime}(x)<0$ for all $1 \leq i \leq n$ and $x \in \mathbb{R}$, then $\left(\bar{u}_{m}\right)_{i}^{\prime}(x)<0$ for all $1 \leq i \leq n$ and $x \in[-m r, m r-2 \delta]$.

Proof. By Theorem 2.2, $\bar{u}_{m}$ satisfies $-(L u)(x)=g(u(x), u(x+\delta))$ on $[-m r, m r-2 \delta]$. That is,

$$
a_{i}\left(\bar{u}_{m}(x)\right)_{i}^{\prime \prime}+c\left(\bar{u}_{m}(x)\right)_{i}^{\prime}=-g_{i}\left(\bar{u}_{m}(x), \bar{u}_{m}(x+\delta)\right), \quad 1 \leq i \leq n .
$$

For a fixed $1 \leq i \leq n$, let $y_{m}=\left(\bar{u}_{m}\right)_{i}^{\prime}$. We claim that $y_{m}(-m r)<0$. This is due to the fact that $\bar{u}_{m}(-m r)=\bar{\rho}(-m r), \bar{u}_{m}(x) \leq \bar{\rho}(x)$ for $x \in[-m r, m r]$ and $\bar{\rho}_{i}^{\prime}(-m r)<0$. Using the positivity of $g$, we have

$$
a_{i} y_{m}^{\prime}(x)+c y_{m}(x)=-g_{i}\left(\bar{u}_{m}(x), \bar{u}_{m}(x+\delta)\right) \leq 0, \quad x \in[-m r, m r-2 \delta] .
$$

Therefore, $y_{m}(-m r)<0$ implies $y_{m}(x)<0$ for $x \in[-m r, m r-2 \delta]$, completing the proof.

Now we extend $\bar{u}_{m}$ and $\underline{u}_{m}$ to the whole real line $\mathbb{R}$ by

$$
\bar{v}_{m}= \begin{cases}\bar{u}_{m} & \text { in }[-m r, m r], \\ \bar{\rho} & \text { in } \mathbb{R} \backslash[-m r, m r]\end{cases}
$$

and

$$
\underline{w}_{m}= \begin{cases}\bar{u}_{m} & \text { in }[-m r, m r], \\ \underline{\rho} & \text { in } \mathbb{R} \backslash[-m r, m r]\end{cases}
$$

Then we have

Lemma 2.5. For $m=1,2, \ldots$, the following hold:

(1) $\underline{\rho} \leq \bar{v}_{m+1} \leq \bar{v}_{m} \leq \bar{\rho}$ in $\mathbb{R}$.

(2) $\underline{\rho} \leq \underline{w}_{m} \leq \underline{w}_{m+1} \leq \bar{\rho}$ in $\mathbb{R}$.

Proof. (1) We only need to prove $\bar{v}_{m+1} \leq \bar{v}_{m}$ in $\mathbb{R}$ for $m=1,2, \ldots$ Since $\bar{v}_{m+1} \in$ $C^{2+\alpha}\left([-(m+1) r,(m+1) r-2 \delta] ; \mathbb{R}^{n}\right) \cap C^{1+\alpha}\left([-(m+1) r,(m+1) r] ; \mathbb{R}^{n}\right)$ satisfies

$$
\left\{\begin{aligned}
&-\left(L \bar{v}_{m+1}\right)(x)= g\left(\bar{v}_{m+1}(x), \bar{v}_{m+1}(x+\delta)\right) \\
& \quad \text { for } x \in[-m r, m r] \subset[-(m+1) r,(m+1) r-2 \delta], \\
& \bar{v}_{m+1}( \pm m r) \leq \bar{\rho}( \pm m r),
\end{aligned}\right.
$$


$\bar{v}_{m+1}$ is actually a lower solution of (2.16) and (2.18). By Theorem 2.2, there exists at least one solution $u^{*} \in C^{2+\alpha}\left([-m r, m r-2 \delta] ; \mathbb{R}^{n}\right) \cap C^{1+\alpha}\left([-m r, m r] ; \mathbb{R}^{n}\right)$ such that $u^{*}$ satisfies (2.16) and (2.18) and $\bar{u}_{m+1}=\bar{v}_{m+1} \leq u^{*} \leq \bar{\rho}$ in $[-m r, m r]$. Now, the maximality of $\bar{u}_{m}$ in the sense of Theorem 2.2 implies that $u^{*} \leq \bar{u}_{m}$ in $[-m r, m r]$, and hence $\bar{v}_{m+1} \leq u^{*} \leq \bar{u}_{m}=\bar{v}_{m}$ in [-mr, $\left.m r\right]$. But, $\bar{v}_{m}=\bar{\rho} \geq \bar{v}_{m+1}$ in $\mathbb{R} \backslash[-m r, m r]$. Therefore, we obtain $\bar{v}_{m+1} \leq \bar{v}_{m}$ in $\mathbb{R}$.

The proof of (2) is similar.

By Lemma 2.5, we know that

$$
\lim _{m \rightarrow \infty} \bar{v}_{m}(x)=\bar{v}(x) \quad \text { and } \quad \lim _{m \rightarrow \infty} \underline{w}_{m}(x)=\underline{w}(x)
$$

exist for each given $x \in \mathbb{R}$, and the convergence is uniform on every bounded interval of $\mathbb{R}$.

Theorem 2.6. Let $\bar{\rho}$ and $\rho$ be a pair of upper and lower solutions of (2.14) satisfying $\underline{\rho} \leq \bar{\rho}$ in $\mathbb{R}$. Then both $\bar{v}$ and $\underline{w}$ are solutions of (2.14) in $\mathbb{R}$.

Proof. We only consider $\bar{v}$, since the proof for $\underline{w}$ is similar.

It suffices to prove that $\bar{v}$ satisfies $(2.16)$ in $[-m r, m r-2 \delta]$ for every fixed large $m$. Let $k \geq m+1$ be an arbitrary integer. Then $\bar{v}_{k}$ satisfies

$$
\left\{\begin{array}{l}
-\left(L \bar{v}_{k}\right)(x)=g\left(\bar{v}_{k}(x), \bar{v}_{k}(x+\delta)\right) \quad \text { in }[-m r, m r] \subset[-k r, k r-2 \delta] \\
\bar{v}_{k}( \pm m r)=\bar{u}_{k}( \pm m r)
\end{array}\right.
$$

By continuity of $g$, we know that

$$
g\left(\bar{v}_{k}(x), \bar{v}_{k}(x+\delta)\right) \rightarrow g(\bar{v}(x), \bar{v}(x+\delta)) \quad \text { uniformly on }[-m r, m r] \text { as } k \rightarrow \infty .
$$

So, if we define $\bar{g}_{k}$ by

$$
\bar{g}_{k}(x)=g\left(\bar{v}_{k}(x), \bar{v}_{k}(x+\delta)\right) \quad \text { in }[-m r, m r],
$$

then the sequence $\left\{\bar{g}_{k}\right\}_{k \geq m+1}$ is bounded in $L_{p}\left([-m r, m r] ; \mathbb{R}^{n}\right)$ for every $p \geq 1$. Therefore, $\left\{\bar{v}_{k}\right\}_{k \geq m+1}$ is bounded in $W_{p}^{2}\left([-m r, m r] ; \mathbb{R}^{n}\right)$ for every $p \geq 0$. Thus the embedding result yields the boundedness of $\left\{\bar{v}_{k}\right\}_{k \geq m+1}$ in $C^{1+\mu}\left([-m r, m r] ; \mathbb{R}^{n}\right)$ $\left(\mu=1-\frac{1}{p}\right)$. Therefore $\left\{\bar{v}_{k}\right\}_{k \geq m+1}$ is bounded in $\bar{C}^{1+\alpha}\left([-m r, m r] ; \mathbb{R}^{n}\right)$. That is, there exists $N_{0}>0$ such that

$$
\begin{aligned}
\left\|\bar{v}_{k}\right\|_{C^{\alpha}\left([-m r, m r-2 \delta] ; \mathbb{R}^{n}\right)} & \leq\left\|\bar{v}_{k}\right\|_{C^{1+\alpha}\left([-m r, m r-2 \delta] ; \mathbb{R}^{n}\right)} \\
& \leq\left\|\bar{v}_{k}\right\|_{C^{1+\alpha}\left([-m r, m r] ; \mathbb{R}^{n}\right)} \leq N_{0}, \quad k \geq m+1 .
\end{aligned}
$$

Reasoning in a similar way to that in Step 2 of the proof of Theorem 2.2, we can then establish the boundedness of $\left\{\bar{v}_{k}\right\}_{k \geq m+1}$ in the $C^{2+\alpha}\left([-m r, m r-2 \delta] ; \mathbb{R}^{n}\right)$ topology. Thus, the well-known Arzela-Ascoli theorem implies that $\left\{\bar{v}_{k}\right\}_{k \geq m+1}$ contains a subsequence which converges in $C^{2}\left([-m r, m r-2 \delta] ; \mathbb{R}^{n}\right)$ to a function $v^{*} \in C^{2+\alpha}\left([-m r, m r-2 \delta] ; \mathbb{R}^{n}\right)$. But $\left\{\bar{v}_{k}\right\}_{k \geq m+1}$ itself converges pointwise to $\bar{v}$ in $[-m r, m r-2 \delta]$. So, $v^{*}$ and $\bar{v}$ must coincide in $[-m r, m r-2 \delta]$. This means that the whole sequence $\left\{\bar{v}_{k}\right\}_{k \geq m+1}$ converges in $C^{2}\left([-m r, m r-2 \delta] ; \mathbb{R}^{n}\right)$ to $v^{*}=\bar{v}$ as $k \rightarrow \infty$. Therefore, $L \bar{v}_{k} \rightarrow L \bar{v}$ pointwise in $[-m r, m r-2 \delta]$ as $k \rightarrow \infty$. By taking 
the limit as $k \rightarrow \infty$ in (2.21) for each $x \in[-m r, m r-2 \delta]$, we get

$$
-(L \bar{v})(x)=g(\bar{v}(x), \bar{v}(x+\delta)) \quad \text { in }[-m r, m r-2 \delta] .
$$

This completes the proof of the theorem.

\section{Proof of the main Result}

Let $c>c^{*}(\tau)$. Then $g_{\tau}(c)<c$ by Lemma 1.1. Definition of $g_{\tau}(c)$ then implies that there exists a $\bar{\rho} \in \Gamma$ such that $\Psi(\bar{\rho}, c, \tau)<c$, i.e.

$$
\sup _{\substack{x \in \mathbb{R} \\ j \in\{1, \ldots, n\}}} \frac{a_{j} \rho_{j}^{\prime \prime}(x)+f_{j}(\rho(x), \rho(x+c \tau))}{-\rho_{j}^{\prime}(x)}<c
$$

which yields

$$
A \bar{\rho}^{\prime \prime}(x)+c \bar{\rho}^{\prime}(x)+f(\bar{\rho}(x), \bar{\rho}(x+c \tau))<0, \quad x \in \mathbb{R} .
$$

This means that $\bar{\rho}$ is an upper solution of (1.3). On the other hand, $\mathbf{0}$ is obviously a lower solution of (1.3) due to (A2). This yields a pair of ordered upper and lower solutions $\mathbf{0}$ and $\bar{\rho}$. By Theorem 2.2 and Lemma 2.4, there exists a sequence of vector functions $\left\{v_{k}\right\}_{k \geq 1}$ satisfying

(i) $v_{k} \in C^{2+\alpha}\left([-k r, k r-2 c \tau] ; \mathbb{R}^{n}\right) \cap C^{1+\alpha}\left([-k r, k r] ; \mathbb{R}^{n}\right)$, where $r=1+2 c \tau>$ 0

(ii) $v_{k}( \pm k r)=\bar{\rho}( \pm k r)$ and $0 \leq v_{k} \leq \bar{\rho}$ in $\mathbb{R}$;

(iii) $\left(v_{k}\right)_{i}^{\prime}<0$ in $[-k r, k r-2 c \tau], 1 \leq i \leq n$.

Furthermore, $v_{k} \rightarrow v$ in $\mathbb{R}$ and $v$ satisfies (1.3) in $\mathbb{R}$ by Theorem 2.6. So, we obtain a solution $v$ of (1.3) which is monotonically decreasing and satisfies $\lim _{x \rightarrow \infty} v(x)=\mathbf{0}$. It remains to show that $\lim _{x \rightarrow-\infty} v(x)=1$. By monotonicity and boundedness of $v$, we know that $\lim _{x \rightarrow \infty} v(x)=q$ exists and $\mathbf{0}<q \leq \mathbf{1}$. It can be shown that $q$ must satisfy $f(q, q)=\mathbf{0}$. But (A2) implies that $\mathbf{0}$ and $\mathbf{1}$ are the only vectors $w \in[\mathbf{0}, \mathbf{1}]$ such that $f(w, w)=0$. This leads to $q=\mathbf{1}$, and therefore completes the proof.

\section{REFERENCES}

1. S. Agmon, A. Douglis and L. Nirenberg, Estimates near the boundary for solutions of elliptic partial differential equations satisfying general boundary conditions, Part I and Part II, Comm. Pure Appl. Math. 12 (1959), 623-727 and 27 (1964), 35-59. MR 23:A2610; MR 28:5252

2. N. F. Britton, Reaction-Diffusion Equations and Their Applications to Biology, Academic, New York, 1986. MR 88k:92001

3. P. C. Fife, Mathematical Aspects of Reaction-Diffusing Systems, Lecture Notes in Biomath., Vol. 28, Springer-Verlag, New York, 1979. MR 80g:35001

4. R. Gardner, Review on Traveling Wave Solutions of Parabolic Systems by Aizik I. Volpert, Vitaly A. Volpert and Vladimir A. Volpert, Bull. Amer. Math. Soc. 32 (1995), 446-452.

5. A. W. Leung, Systems of Nonlinear Partial Differential Equations with Applications to Biology and Engineering, Kluwer, Dordrecht, 1989.

6. J. D. Murray, Mathematical Biology, Springer-Verlag, New York, 1989. MR 90g:92001

7. C. V. Pao, Nonlinear Parabolic and Elliptic Equations, Plenum, New York, 1992. MR 94c:35002 
8. K. W. Schaaf, Asymptotic behavior and traveling wave solutions for parabolic functional differential equations, Trans. Amer. Soc. 302 (1987), 587-615. MR 88g:35190

9. A. I. Volpert, V. A. Volpert and V. A. Volpert, Traveling Wave Solutions of Parabolic Systems, Transl. Math. Monographs, Vol. 140, Amer. Math. Soc., Providence, RI, 1994. MR 96c:35092

Department of Mathematics and Statistics, York University, North York, Ontario, CANADA M3J 1P3

E-mail address: xzou@mathstat.yorku.ca

Current address: Department of Mathematics and Statistics, University of Victoria, British Columbia, Canada V8W 3P4

E-mail address: xzou@math.uvic.ca

E-mail address: wujh@mathstat.yorku.ca 